\title{
Pancreatic neuroendocrine tumours: spectrum of imaging findings
}

\author{
Eleonora Bicci ${ }^{1}$, Diletta Cozzi ${ }^{1}$, Riccardo Ferrari ${ }^{2}$, Giulia Grazzini ${ }^{1}$, Silvia Pradella $^{1}$, Vittorio Miele ${ }^{1}$ \\ ${ }^{1}$ Department of Emergency Radiology, Careggi University Hospital, Florence, Italy; ${ }^{2}$ Department of Emergency Radiology, San Camillo Forlanini \\ Hospital, Rome, Italy \\ Contributions: (I) Conception and design: E Bicci, D Cozzi, V Miele; (II) Administrative support: S Pradella, V Miele, R Ferrari; (III) Provision of \\ study materials or patients: E Bicci, D Cozzi, G Grazzini; (IV) Collection and assembly of data: None; (V) Data analysis and interpretation: None; (VI) \\ Manuscript writing: All authors; (VII) Final approval of manuscript: All authors. \\ Correspondence to: Diletta Cozzi. Department of Emergency Radiology, Careggi University Hospital, 50134 Florence, Italy. \\ Email: dilettacozzi@gmail.com.
}

\begin{abstract}
Pancreatic neuroendocrine tumours (pNETs) are rare and heterogeneous group of neoplasms presenting with a wide variety of symptoms and biological behaviour, from indolent to aggressive ones. pNETs are stratified into functional or non-functional, because of their ability to produce metabolically active hormones. pNETs can be an isolate phenomenon or a part of a hereditary syndrome like von HippelLindau syndrome or neurofibromatosis-1. The incidence has increased in the last years, also because of the improvement of cross-sectional imaging. Computed tomography (CT), magnetic resonance imaging (MRI) and functional imaging are the mainstay imaging modalities used for tumour detection and disease extension assessment, due to easy availability and better contrast/spatial resolution. Radiological imaging plays a fundamental role in detection, characterization and surveillance of pNETs and is involved in almost every stage of patients' management. Moreover, with specific indications and techniques, interventional radiology can also play a role in therapeutic management. Surgery is the treatment of choice, consisting of either partial pancreatectomy or enucleation of the primary tumour. This article reviews the radiologic features of different pNETs as well as imaging mimics, in order to help radiologists to avoid potential pitfalls, to reach the correct diagnosis and to support the multidisciplinary team in establishing the right treatment.
\end{abstract}

Keywords: Neuroendocrine tumour (NET); computed tomography (CT); magnetic resonance; radiomics

Submitted May 30, 2020. Accepted for publication Sep 09, 2020.

doi: $10.21037 /$ gs-20-537

View this article at: http://dx.doi.org/10.21037/gs-20-537

Pancreatic neuroendocrine tumours (pNETs) are rare tumours that arise from the neuroendocrine cells of the pancreas (1). They are less than $3 \%$ of pancreatic tumours, with a growing annual incidence of one case per 100,000 individuals (1-3). pNETs can be an isolate phenomenon or a part of a hereditary syndrome like multiple endocrine neoplasia type 1 (MEN1), von Hippel-Lindau syndrome or neurofibromatosis type 1 (4). pNETs can be malignant neoplasms (5). Because of their ability to secrete hormones, pNETs are stratified into functional or non-functional ones (6). The WHO classification from 2017, only for pNETs, divides them into well-differentiated and poorly differentiated (also named as neuroendocrine carcinomas), and the former is further subdivided into grade $1(\mathrm{G} 1)$, grade 2 (G2) and grade 3 (G3) (7). This grading refers to the mitotic rate and $\mathrm{Ki}-67$ index suggesting the malignant potential of these tumours $(8,9)$. The histological grade of a pNET is strongly connected with long-term survival, symbolizing the malignant nature of the tumour, and is having an important impact on therapy (1). Surgery is recommended as treatment of choice for small and localized pNETs, with a high survival rate $(10,11)$. For the diagnosis and characterization both laboratory testing (serum markers such as Chromogranin A and hormones produced by functioning NET) and multimodality imaging are useful $(12,13)$. Accurate knowledge of the potential and applications of imaging modalities, both standard and advanced, is essential for an accurate study of abdominal 

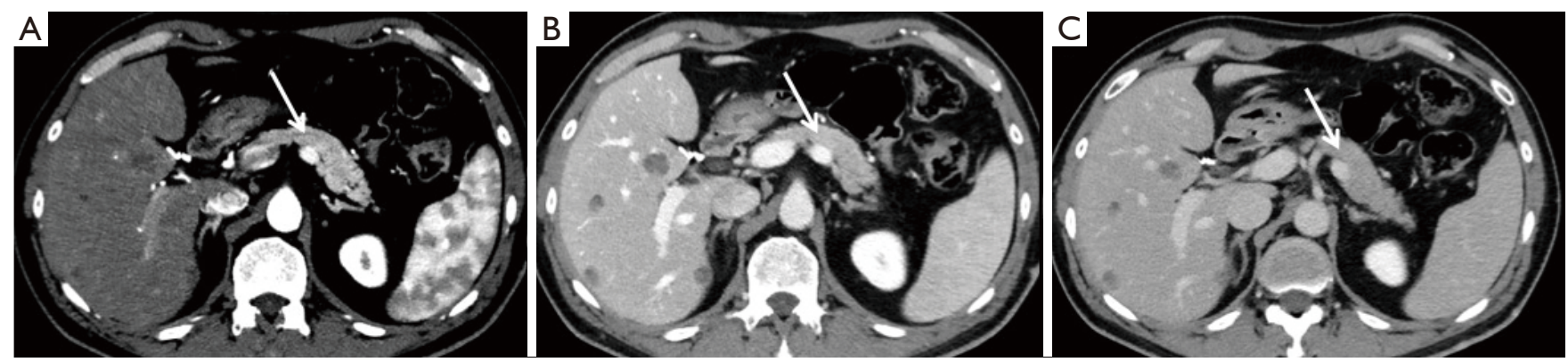

Figure 1 Hypervascular pNET with hypovascular metastases. CT images in (A,B,C) show a hyperenhancing pancreatic nodule in the bodytail of the organ (arrows). Please note the atypical hepatic metastases in the right lobe that are hypovascular in arterial, pancreatic and venous phase. pNET, pancreatic neuroendocrine tumour; CT, computed tomography.

oncological pathology either for the initial assessment, the treatment planning, and the follow-up after therapy (14). A lot of imaging methods can be useful including ultrasound (US), computed tomography (CT), magnetic resonance imaging (MRI) and functional/nuclear imaging such as somatostatin receptor imaging and positron emission tomography (PET). The association between PET and CT (PET/CT) with different tracers can be particularly important during staging and search of metastases (15). Radiological imaging plays a fundamental contributory role in detection, characterization and surveillance of pNETs and is involved in almost every stage of patients' management and can be useful both for diagnosis and surgical or medical therapy. Moreover, with specific indications and techniques, interventional radiology can also play a role in therapeutic management $(16,17)$.

In this article, we reviewed the different radiological findings at CT and MRI related to the different patterns of pNETs. Radiologists should be aware of these potential accidental findings and collaborate with clinicians to reach an accurate diagnosis and to decide the right therapy. We present the following article in accordance with the Narrative Review reporting checklist (available at http:// dx.doi.org/10.21037/gs-20-537).

\section{Non-functioning tumours}

Non-functioning tumours are $60-80 \%$ of pNETs and approximately $50-90 \%$ of them are malignant at presentation also because they tend to manifest late and often larger in size $(1,18)$. Abdominal US has low sensitivity and specificity for detecting pancreatic solid nodules or masses but can help in finding liver metastases $(19,20)$.
Recently, contrast-enhanced US (CEUS) improves the accuracy of analysis of focal pancreatic lesions through the use of US contrast medium (20). In fact, the bloody supply to the pancreas is entirely arterial and enhancement of the gland is well recognized through CEUS examination: similarly to CT exam as explained below, also pancreatic CEUS shows a hyperenhancing arterial phase in pNETs (21). CT is the imaging method of choice for the evaluation of various pancreatic diseases and is often the initial diagnostic technique, also because of its high spatial resolution and short acquisition time (22). Both an arterial angiographic phase, after 20-30 seconds, or a pancreatic one, 20 seconds later than the first, can be used, followed, after $60-80$ seconds by a portal-venous phase $(23,24)$. The arterial phase is considered the gold standard in localizing pNETs, as they are usually hypervascular, and because of their contrast washout in the venous phase, that makes the tumour isodense respect to the surrounding parenchyma. Referring to this particular behaviour, pNETs tend to be a well-defined and homogeneously enhanced lesions on arterial-phase CT, due to their copious capillary pathway, and hypo-iso or hyperdense on portal phase, in contrast with pancreatic parenchyma (Figure 1) (25). A lot of research has been made to search a way to differentiate tumour grades both with CT and MRI (26,27). Zamboni et al. in their retrospective study, tried to differentiate various grades of pNETs in 148 patients using CT, and demonstrated that the parameters with better performance in G1-G2 tumours were the hypervascular aspect in the arterial phase, hyperdensity in the venous phase and well-defined margins. On the other hand, they affirmed that G3 tumours are larger ones, non-hypervascular in the arterial phase and hypodense in the venous phase and tend to be more invasive (26). 

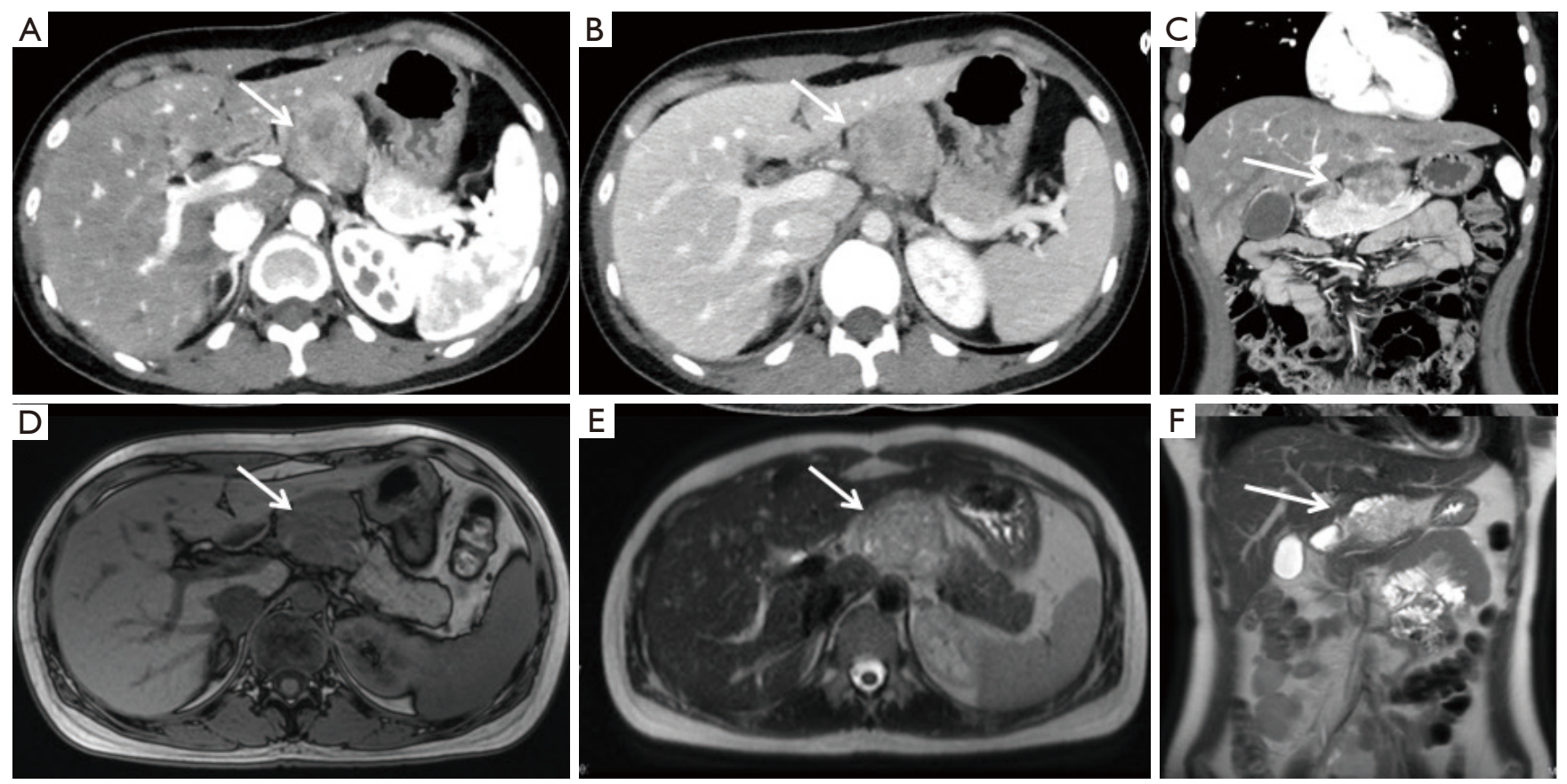

Figure 2 CT and MRI imaging. A large pNET (arrows) in a young woman that underwent a CT exam [(A) axial arterial, (B) axial portal phases and (C) coronal arterial reconstruction] and MRI [(D) T1-weighted, (E) T2-weighted imaging and (F) coronal T2 reconstruction]. Note the non-homogeneous vascularization of the lesion and the relatively hyperintensity in T2 weighted phase. CT, computed tomography; MRI, magnetic resonance imaging; pNET, pancreatic neuroendocrine tumour.

A similar study was made by Takumi et al. evaluating multiple parameters such as tumour's margin, presence of peripancreatic vascular involvement, ductal dilatation, metastasis (lymph nodal $\mathrm{N}$ or distant ones $\mathrm{M}$ ), cystic or necrotic aspects. They found that G1 and G2 have no great difference referring to tumour morphology, vascular involvement, ductal dilatation, or $\mathrm{N}$ involvement; instead, G2 are more often larger in size $(\geq 20 \mathrm{~mm})$ and show no hyper-attenuation during the portal phase (28). It is easy to understand from these studies how limited is the possibility to differentiate G1 from G2, being only few radiological characteristics related with tumour grade.

With the advent of Radiomics, the new method in which the information is "hidden" inside the radiological images and can be extracted using advanced texture and shape analysis, it has been possible to extract lots of information regarding the structure we need to study (29-31). Zhao et al. tried to overcome these limits using radiomics features on CT images to identify some discriminant features for G1 and G2 tumours: he found that six features, using both non-enhanced and portal-venous phases, can be useful in differentiating grade G1/G2 in non-functional pNETs (32).
More information in the characterization of these different grades can be obtained with MRI imaging (15). The pancreas has a hyperintense signal on T1-weighted imaging, because of the amount of protein inside the parenchyma, therefore pNETs tend to appear hypointense in contrast with pancreatic parenchyma on un-enhanced T1-weighted images. On T2-weighted images, pNETs can be hyperintense, even if a lower signal can also be present. On post-contrast images, tumours show vivid contrast enhancement, that can be both homogeneous or heterogeneous, ring- or target-like (Figure 2). Diffusion weighted imaging (DWI) may also help in tumour detection $(33,34)$. Guo et al. studied a total of 59 lesions trying to identify the MRI features in differentiating pNETs G1/ G2 and pancreatic neuroendocrine carcinoma G3; G1/G2 tumours had well-circumscribed border compared with G3 (35). G3 tend to be bigger in size, often associated with metastases and duct dilatation, low-moderate enhancement also because of necrotic phenomena, and high DWI signal intensity, with lower apparent diffusion coefficient (ADC) values than G1/G2 (35). They concluded that the presence of metastases and the different ADC value between grades 

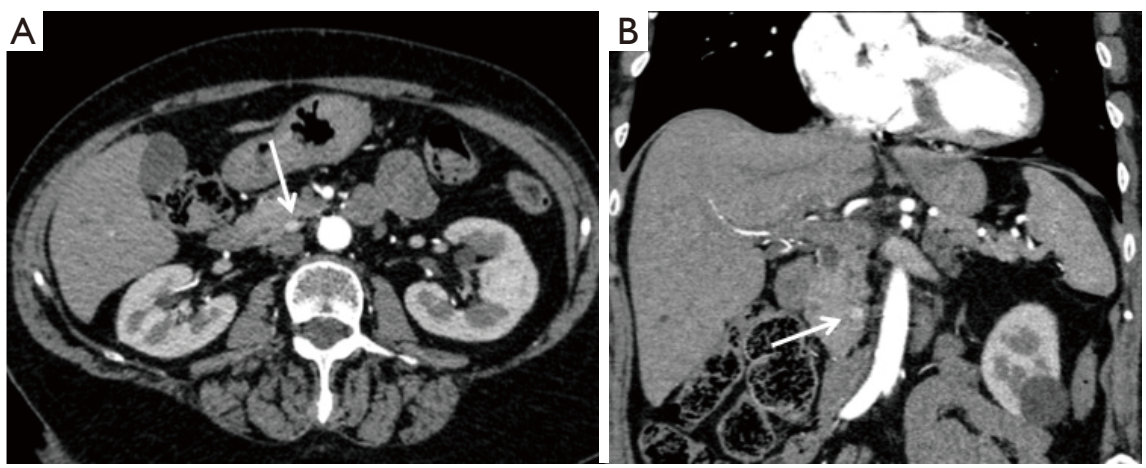

Figure 3 Insulinoma. Pancreatic nodular lesion smaller than $1 \mathrm{~cm}$ in the uncinate process (arrows). CT arterial phase in axial (A) and coronal (B) reconstruction. CT, computed tomography.

can be useful in distinguishing G3 from G1/G2 pNETs. Also, Mebis et al. tried to find the connection between ADC value on MRI and histopathologic WHO-grades of NET. They found interesting and important differences between low (G1-G2), with higher values of ADC, and high grade (G3) pNETs (36). The lower ADC values on ADC map in high grade pNETs may be caused by the high cellularity with less extracellular space and cytoplasm, reducing the possibilities of water molecules to move (37). Also, fibrosis can be involved in generating lower $\mathrm{ADC}$ values, above all in some low grade, well-differentiated tumours (38). De Robertis et al. tried to improve the use of MRI in association with histogram analysis parameters finding that, in the analysis of 42 pNETs $>1 \mathrm{~cm}$, the whole-tumour histogram analysis of ADC maps may be used as a predictor of tumour grade, vascular invasion and nodal or liver metastases. Furthermore, ADC entropy and ADC kurtosis resulted as the most significant parameters in discerning tumours with malignant behaviour (39).

\section{Functioning tumours}

pNETs can be hyperfunctioning, with the production of a lot of different hormones such as insulin, glucagon, gastrin, vasoactive intestinal peptide (VIP) and somatostatin, that can create various clinical manifestation. This subtype of tumours tends to present earlier, with clinical signs and symptoms. Insulinomas are the most common functioning NET, representing the $40 \%$ of all functioning pNETs (40). Typically, insulinomas are located equally in all the anatomic sites of the pancreas, and they can be round-shaped, nodular or oval, usually with a diameter inferior to $15 \mathrm{~mm}$. They can be isolate or multiple and both deep or superficial (41).
At CT examination, these lesions are isodense at baseline, homogeneously hyperdense in the pancreatic phase, and hypodense in the venous one because of their washout phenomena (Figure 3). These aspects where confirmed by the study that included 53 patients with insulinoma, conducted by Fidler $e t$ al., demonstrating that most tumours were hyperenhancing compared with the pancreatic parenchyma on at least one of the phases; nevertheless three tumours were hypoattenuating on all phases and another three were isodense within the pancreatic parenchyma, but could be visualized because of their pedunculated morphology (42). In order to distinguish between typical and atypical insulinomas, multiphase imaging is essential: the latter is iso-hypoattenuating in the early phase at CT, and hypoattenuating in venous one, because of its higher stromal composition, its smaller dimensions and its structure with both amyloid and fibrohyaline components (43). MRI shows a homogeneously enhanced lesion with low signal on T1-weighted imaging and high signal on T2weighted one (44). In their case report of a female patient with a clinical suspicion of insulinoma and an inconclusive contrast-enhanced CT and MRI, Anaye et al. underlined the usefulness of DWI sequences in detecting and localizing small insulinomas especially for those with no hypervascular pattern, with high signal intensity on DWI. Furthermore, ADC in that area was reduced as compared to the normal pancreatic parenchyma (45).

Gastrinomas are the second most common functioning pNETs. Their location is usually in the 'gastrinoma triangle' (90\%) that is a virtual space marked superiorly by the cystic and common ducts, inferiorly by the 2 nd and 3 rd parts of the duodenum and medially by the connection between the neck and body of the pancreas (46). These tumours tend to 

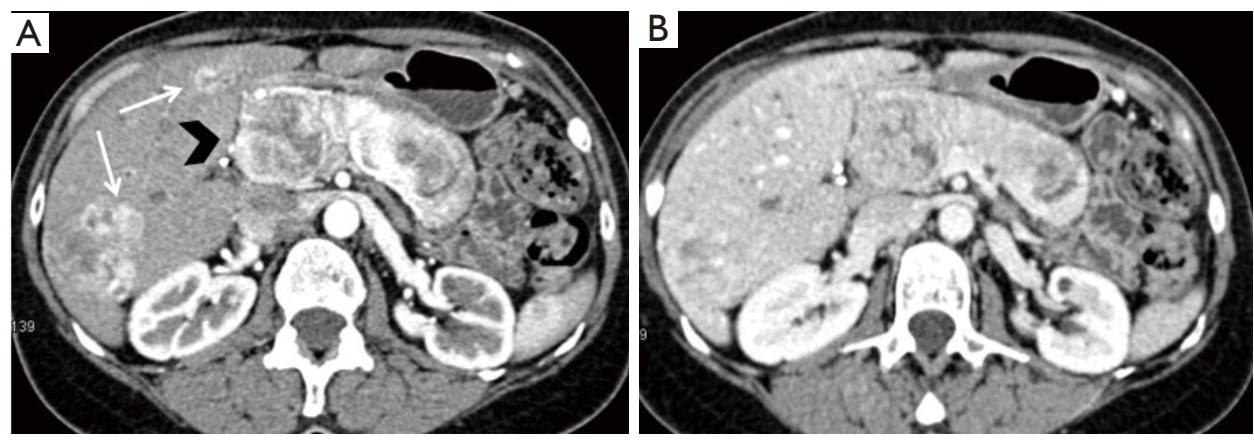

Figure 4 Hypervascular liver metastases. These axial CT images show a huge pNET (black arrowhead) with non-homogeneous arterial (A) and venous (B) enhancement. Liver metastases (white arrows) show the same dynamic contrast enhancement of the primary pancreatic lesion. CT, computed tomography; pNET, pancreatic neuroendocrine tumour.

be smaller in size $(0.3-3 \mathrm{~cm})$, less vascular and with a higher probability than insulinomas to be extra-pancreatic (47). At CT examination, on baseline images they are hypodense, hyperattenuating in early enhanced phase and hypodense in the venous one. At MRI they show low signal intensity in T1-weighted images, high signal intensity in T2-weighted ones and enhancement in the pancreatic phase (48). In the study conducted by Semelka et al. analysing 22 pNETs, they found that gastrinomas are often different in appearance than other NETs as they usually have a ring-enhancement, whereas non-gastrinoma-non-insulinoma tumours usually enhance heterogeneously (49).

The others subtype of functioning pNETs represent $5 \%$ of this class, including glucagonoma, somatostatinoma, VIPoma, ACTHoma and PPoma. These functioning tumours have a low incidence and may show different clinical manifestations (47). They tend to be isolate lesions, larger than insulinomas, with non-specific and heterogeneous enhancement pattern that can be both hypodense or hyperdense, due to necrosis or haemorrhagic aspects. On MRI, they usually have low signal intensity on T1-weighted images and high signal intensity on T2-weighted ones. The article by Sofka et al. about a $2 \mathrm{~cm}$ VIPoma of the pancreatic tail demonstrated a moderate signal intensity of the lesion in T2- weighted images, suggesting that these tumours may have different aspects on T2-weighted sequences (50). Because of these variable imaging findings that may be present, above all for small hyperfunctioning ones, it seems that DWI may better depict and characterize small pNETs, due to its greater image contrast and functional information $(51,52)$. Farchione et al. in their study affirmed that
DWI will be particularly useful in those patients with clinical suspicion for pNETs and with negative or suspicious conventional imaging findings, or in those with contraindications to contrast medium injection (53). Brenner et al. found that using both high b-value diffusionweighted and T2-weighted MR images improve detection of pNETs relative to either technique alone (54).

\section{Metastasis and mimics}

Non-functional pNETs are usually malignant (90\%), approximately $50-60 \%$ of gastrinomas show these characteristics, whereas $90 \%$ of insulinomas are benign (55). They can generate metastases above all in lymph nodes, bones and liver (Figure 4). Hepatic metastases usually show hypointensity or isointensity on T1-weighted imaging in comparison with the surrounding parenchyma, hyperintensity on T2-weighted sequences. In some rare cases they can have a different presentation with both hyperintensity on $\mathrm{T} 1$ and hypointensity on T2-weighted images. The same characteristics may be seen at CT. Also baseline imaging is very important in the evaluation of these lesions, as their enhancement is usually higher during the arterial phase (Figure 5) (56). On the other hand, in the study of Armstrong et al. on 51 patients who underwent triple phase CT, 23 demonstrated hypoenhancement pattern, 18 a mixed one and only 10 showed a hyperenhancement in arterial phase (Figure 1) (57).

Bone metastases frequently involved axial skeleton. They can show both osteolytic or osteosclerotic aspects at traditional radiography; $10 \%$ are purely osteolytic (58). They are not uncommon, observed in $42 \%$ of patients at 

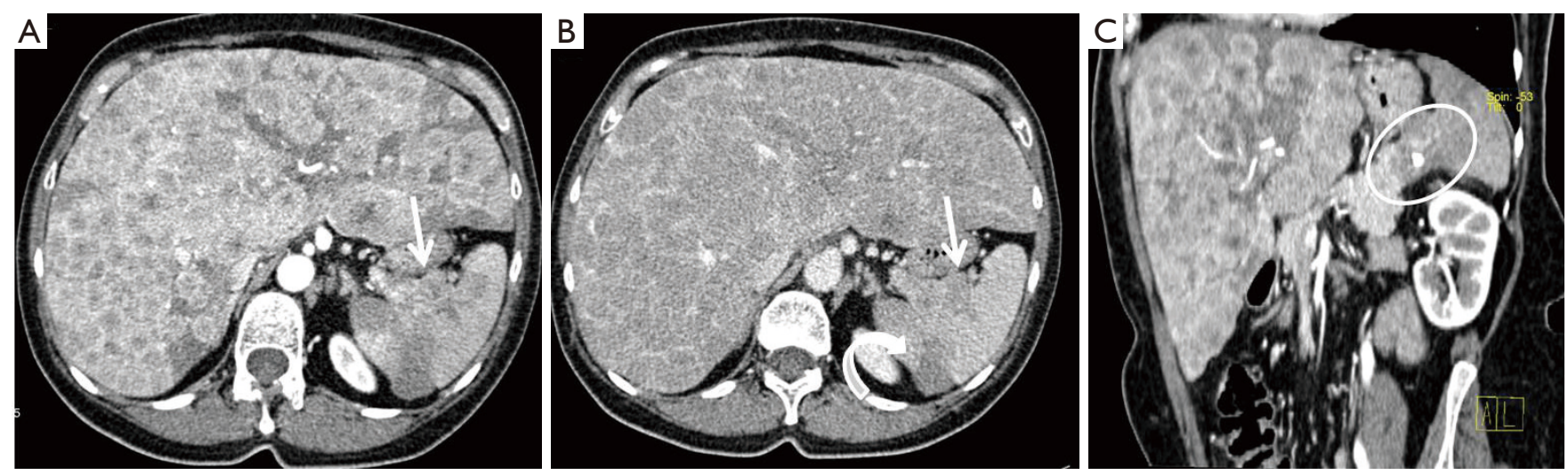

Figure 5 Pancreatic tail pNET. These CT images [(A) axial arterial, (B) axial venous phase and (C) multiplanar reconstruction] show a pancreatic tail pNET (arrows) relatively hypodense, with non-regular margins, that infiltrates splenic hilum and vessels and cause segmental splenic infarct (curved arrow). Liver parenchyma is diffusely interested by multiple nodular metastases. pNET, pancreatic neuroendocrine tumour; CT, computed tomography.

Table $1 \mathrm{pNETs}$ mimics and their main differential diagnosis

\begin{tabular}{ll}
\hline Mimics & Differential diagnosis \\
\hline Metastases & Usually metastases from RCC: hypervascular lesions in patient with history of RCC \\
Intrapancreatic splenule & $\begin{array}{l}\text { Usually in the pancreas tail: nodule with the same enhancing phases of splenic } \\
\text { parenchyma }\end{array}$ \\
Mostly-solid serous cystoadenoma & Non distinguishable from pNETs; further investigations needed \\
GISTs & $\begin{array}{l}\text { Mass from the stomach or duodenum; fat usually separates GIST from pancreatic } \\
\text { parenchyma }\end{array}$ \\
Peripancreatic paraganglioma & A mass that does not come from pancreatic parenchyma (interposed fatty tissue) \\
\hline
\end{tabular}

RCC, renal cell carcinoma; GIST, gastrointestinal stromal tumour.

autopsy (59). Lesions are hypointense on T1-weighted images. Even if their predominant aspect is osteoblastic, they can show hyperintense and heterogeneous signal on T2-weighted images.

The differential diagnosis with pNETs, involve some other lesions (Table 1). For example, the metastases from renal cell carcinoma (RCC), are usually hyper-vascular and can be similar to the NETs (55). Gastrointestinal stromal tumours (GISTs), because of their position, arising from stomach or duodenum, and with their vivid contrast enhancement on arterial phase, with both cystic or necrotic phenomena are not easily discernible from a primary pancreatic tumour $(9,60,61)$. pNETs can occasionally manifest as a primarily cystic tumours (for example mostly-solid serous cystadenoma) and differential diagnosis from other cystic neoplasms can be made through the evidence of their hypervascular rim (Figure 6) (62). It has to be remembered also that accessory spleens are commonly located in the pancreatic tail and with their high enhancement it can be difficult to distinguish them from pNETs (63). Zhang et al. reported two rare cases of paragangliomas of the pancreas, which are typically hypervascular, with cystic changes mimicking pNETs (64). Furthermore, especially non-functioning pNETs that are bigger in size, may invade surrounding structures and present with biliary obstruction, as the classic presentation for pancreatic adenocarcinomas (65-68).

\section{Conclusions}

pNETs are rare tumours but their incidence is growing because of our better radiological detection especially 

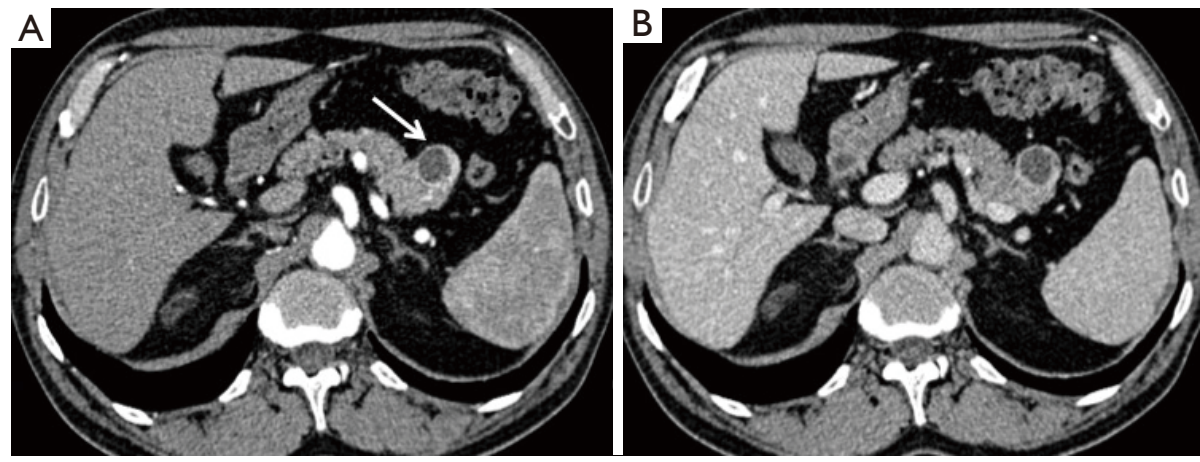

Figure 6 pNET mimics a cystic neoplasm. A round partially cystic neoplasm of the pancreatic tail with a hypervascular rim that is typical of a pNET [arrow in (A)]. Venous phase in (B). pNET, pancreatic neuroendocrine tumour.

through CT and MRI. The management of patients with pNET is multidisciplinary and often multimodal: this underlines the importance of the radiologists as a fundamental figure that must be aware of the typical/ atypical imaging findings or mimics of pNETs, according to up-to-date literature, in order to facilitate the initial diagnosis and to help in the management in patients with such pancreatic lesions.

\section{Acknowledgments}

Funding: None.

\section{Footnote}

Provenance and Peer Review: This article was commissioned by the Guest Editor (Antonio Barile) for the series "Multimodality Advanced Imaging and Intervention in Gland Diseases" published in Gland Surgery. The article has undergone external peer review.

Reporting Checklist: The authors have completed the Narrative Review reporting checklist. Available at http:// dx.doi.org/10.21037/gs-20-537

Conflicts of Interest: All authors have completed the ICMJE uniform disclosure form (available at http://dx.doi. org/10.21037/gs-20-537). The series "Multimodality Advanced Imaging and Intervention in Gland Diseases" was commissioned by the editorial office without any funding or sponsorship. The authors have no other conflicts of interest to declare.

Ethical Statement: The authors are accountable for all the aspects of the work in ensuring that questions related to the accuracy or integrity of any part of the work are appropriately investigated and resolved.

Open Access Statement: This is an Open Access article distributed in accordance with the Creative Commons Attribution-NonCommercial-NoDerivs 4.0 International License (CC BY-NC-ND 4.0), which permits the noncommercial replication and distribution of the article with the strict proviso that no changes or edits are made and the original work is properly cited (including links to both the formal publication through the relevant DOI and the license). See: https://creativecommons.org/licenses/by-nc-nd/4.0/.

\section{References}

1. Belousova E, Karmazanovsky G, Kriger A, et al. Contrast-enhanced MDCT in patients with pancreatic neuroendocrine tumours: correlation with histological findings and diagnostic performance in differentiation between tumour grades. Clin Radiol 2017;72:150-8.

2. Paiella S, Impellizzeri H, Zanolin E, et al. Comparison of imaging-based and pathological dimensions in pancreatic neuroendocrine tumors. World J Gastroenterol 2017;23:3092-8.

3. Fraenkel M, Kim MK, Faggiano A, et al. Epidemiology of gastroenteropancreatic neuroendocrine tumours. Best Pract Res Clin Gastroenterol 2012;26:691-703. 
4. Klöppel G. Classification and pathology of gastroenteropancreatic neuroendocrine neoplasms. Endocr Relat Cancer 2011;18 Suppl 1:S1-16.

5. Choi TW, Kim JH, Yu MH, et al. Pancreatic neuroendocrine tumor: prediction of the tumor grade using CT findings and computerized texture analysis. Acta Radiol 2018;59:383-92.

6. Wu J, Sun C, Li E, et al. Non-functional pancreatic neuroendocrine tumours: emerging trends in incidence and mortality. BMC Cancer 2019;19:334.

7. Lloyd RV, Osamura RY, Klöppel G, et al. editors. WHO classification of tumours of endocrine organs. 4th ed. Lyon: IARC, 2017.

8. Kim DW, Kim HJ, Kim KW, et al. Neuroendocrine neoplasms of the pancreas at dynamic enhanced CT: comparison between grade 3 neuroendocrine carcinoma and grade 1/2 neuroendocrine tumour. Eur Radiol 2015;25:1375-83

9. Grazzini G, Danti G, Cozzi D, et al. Diagnostic imaging of gastrointestinal neuroendocrine tumours (GI-NETs): relationship between MDCT features and 2010 WHO classification. Radiol Med 2019;124:94-102.

10. Wong KP, Tsang JS, Lang BH. Role of surgery in pancreatic neuroendocrine tumor. Gland Surg 2018;7:36-41.

11. Teo RYA, Goh BKP. Surgical resection of pancreatic neuroendocrine neoplasm by minimally invasive surgerythe robotic approach? Gland Surg 2018;7:1-11.

12. Liu Y, Wang Y, Tang W, et al. Multiparametric MR imaging detects therapy efficacy of radioactive seeds brachytherapy in pancreatic ductal adenocarcinoma xenografts. Radiol Med 2018;123:481-8.

13. Agostini A, Borgheresi A, Mari A, et al. Dual-energy CT: theoretical principles and clinical applications. Radiol Med 2019;124:1281-95.

14. Petralia G, Padhani AR, Pricolo P, et al. Wholebody magnetic resonance imaging (WB-MRI) in oncology: recommendations and key uses. Radiol Med 2019;124:218-33.

15. Seith F, Schraml C, Reischl G, et al. Fast non-enhanced abdominal examination protocols in PET/MRI for patients with neuroendocrine tumors (NET): comparison to multiphase contrast-enhanced PET/CT. Radiol Med 2018;123:860-70.

16. Compagnone G, Padovani R, D'Avanzo MA, et al. Summary of the Italian inter-society recommendations for radiation protection optimization in interventional radiology. Radiol Med 2018;123:378-84.
17. Ierardi AM, Biondetti P, Coppola A, et al. Percutaneous microwave thermosphere ablation of pancreatic tumours. Gland Surg 2018;7:59-66.

18. Jarufe NP, Coldham C, Orug T, et al. Neuroendocrine tumours of the pancreas: predictors of survival after surgical treatment. Dig Surg 2005;22:157-62.

19. Hashimoto S, Hirooka Y, Kawabe N, et al. Role of transabdominal ultrasonography in the diagnosis of pancreatic cystic lesions. J Med Ultrason (2001) 2020;47:389-99.

20. Tedesco G, Sarno A, Rizzo G, et al. Clinical use of contrast-enhanced ultrasound beyond the liver: a focus on renal, splenic, and pancreatic applications. Ultrasonography 2019;38:278-88.

21. Xu M, Li XJ, Zhang XE, et al. Application of contrastenhanced ultrasound in the diagnosis of solid pseudopapillary tumors of the pancreas: imaging findings compared with contrast-enhanced computed tomography. J Ultrasound Med 2019;38:3247-55.

22. Danti G, Addeo G, Cozzi D, et al. Relationship between diagnostic imaging features and prognostic outcomes in gastrointestinal stromal tumors (GIST). Acta Biomed 2019;90:9-19.

23. Foti G, Malleo G, Faccioli N, et al. Characterization of adrenal lesions using MDCT wash-out parameters: diagnostic accuracy of several combinations of intermediate and delayed phases. Radiol Med 2018;123:833-40.

24. Grassedonio E, Toia P, La Grutta L, et al. Role of computed tomography and magnetic resonance imaging in local complications of acute pancreatitis. Gland Surg 2019;8:123-32.

25. Singh A, Hines JJ, Friedman B. Multimodality imaging of the pancreatic neuroendocrine tumors. Semin Ultrasound CT MR 2019;40:469-82.

26. Zamboni GA, Ambrosetti MC, Zivelonghi C, et al. Solid non-functioning endocrine tumors of the pancreas: correlating computed tomography and pathology. HPB (Oxford) 2017;19:986-91

27. Li WX, Miao F, Xu XQ, et al. Pancreatic Neuroendocrine Neoplasms: CT Spectral Imaging in Grading. Acad Radiol 2020. [Epub ahead of print]. doi: 10.1016/ j.acra.2020.01.033

28. Takumi K, Fukukura Y, Higashi M, et al. Pancreatic neuroendocrine tumors: Correlation between the contrastenhanced computed tomography features and the pathological tumor grade. Eur J Radiol 2015;84:1436-43.

29. Parekh V, Jacobs MA. Radiomics: a new application from established techniques. Expert Rev Precis Med Drug Dev 
2016;1:207-26.

30. Grassi R, Miele V, Giovagnoni A. Artificial intelligence: a challenge for third millennium radiologist. Radiol Med 2019;124:241-2.

31. Abdollahi H, Mofid B, Shiri I, et al. Machine learningbased radiomic models to predict intensity-modulated radiation therapy response, Gleason score and stage in prostate cancer. Radiol Med 2019;124:555-67.

32. Zhao Z, Bian Y, Jiang H, et al. CT-radiomic approach to predict g1/2 nonfunctional pancreatic neuroendocrine tumor. Acad Radiol 2020;27:e272-81.

33. Beyhan M, Sade R, Koc E, et al. The evaluation of prostate lesions with IVIM DWI and MR perfusion parameters at 3T MRI. Radiol Med 2019;124:87-93.

34. Yildiz S, Aralasmak A, Yetis H, et al. MRI findings and utility of DWI in the evaluation of solid parathyroid lesions. Radiol Med 2019;124:360-7.

35. Guo C, Chen X, Xiao W, et al. Pancreatic neuroendocrine neoplasms at magnetic resonance imaging: comparison between grade 3 and grade 1/2 tumors. Onco Targets Ther 2017;10:1465-74.

36. Mebis W, Snoeckx A, Corthouts B, et al. Correlation between apparent diffusion coefficient value on MRI and histopathologic WHO grades of neuroendocrine tumors. J Belg Soc Radiol 2020;104:7.

37. Pereira JA, Rosado E, Bali M, et al. Pancreatic neuroendocrine tumors: correlation between histogram analysis of apparent diffusion coefficient maps and tumor grade. Abdom Imaging 2015;40:3122-8.

38. Wang Y, Chen ZE, Yaghmai V, et al. Diffusion-weighted MR imaging in pancreatic endocrine tumors correlated with histopathologic characteristics. J Magn Reson Imaging 2011;33:1071-9.

39. De Robertis R, Maris B, Cardobi N, et al. Can histogram analysis of MR images predict aggressiveness in pancreatic neuroendocrine tumors? Eur Radiol 2018;28:2582-91.

40. Hruban RH, David S. Klimstra DS. Tumors of the pancreas. AFIP Atlas of Tumor Pathology. 4th ed. Washington, DC: American Registry of Pathology, 2007.

41. Rockall AG, Reznek RH. Imaging of neuroendocrine tumours (CT/MR/US). Best Pract Res Clin Endocrinol Metab 2007;21:43-68.

42. Fidler JL, Fletcher JG, Reading CC, et al. Preoperative detection of pancreatic insulinomas on multiphasic helical CT. AJR Am J Roentgenol 2003;181:775-80.

43. Tamm EP, Kim EE, Ng CS. Imaging of neuroendocrine tumors. Hematol Oncol Clin North Am 2007;21:40932; vii.
44. Owen NJ, Sohaib SA, Peppercorn PD, et al. MRI of pancreatic neuroendocrine tumours. Br J Radiol 2001;74:968-73.

45. Anaye A, Mathieu A, Closset J, et al. Successful preoperative localization of a small pancreatic insulinoma by diffusion-weighted MRI. JOP 2009;10:528-31.

46. Alexander HR Jr. Pancreatic endocrine tumors. Cancer Principles and Practice of Oncology. Philadelphia: Lippincott/Raven, 2001:1788-813.

47. Doppman JL, Shawker TH, Miller DL. Localization of islet cell tumors. Gastroenterol Clin North Am 1989;18:793-804.

48. Rha SE, Jung SE, Lee KH, et al. CT and MR imaging findings of endocrine tumor of the pancreas according to WHO classification. Eur J Radiol 2007;62:371-7.

49. Semelka RC, Custodio CM, Cem Balci N, et al. Neuroendocrine tumors of the pancreas: spectrum of appearances on MRI. J Magn Reson Imaging 2000;11:141-8.

50. Sofka CM, Semelka RC, Marcos HB, et al. MR imaging of metastatic pancreatic VIPoma. Magn Reson Imaging 1997;15:1205-8.

51. Bakir B, Salmaslioglu A, Poyanli A, et al. Diffusion weighted MR imaging of pancreatic islet cell tumors. Eur J Radiol 2010;74:214-20.

52. Caramella C, Dromain C, De Baere T, et al. Endocrine pancreatic tumours: which are the most useful MRI sequences? Eur Radiol 2010;20:2618-27.

53. Farchione A, Rufini V, Brizi MG, et al. Evaluation of the added value of diffusion-weighted imaging to conventional magnetic resonance imaging in pancreatic neuroendocrine tumors and comparison with 68Ga-DOTANOC positron emission tomography/computed tomography. Pancreas 2016;45:345-54.

54. Brenner R, Metens T, Bali M, et al. Pancreatic neuroendocrine tumor: added value of fusion of T2weighted imaging and high b-value diffusion-weighted imaging for tumor detection. Eur J Radiol 2012;81:e746-9.

55. Low G, Panu A, Millo N, et al. Multimodality imaging of neoplastic and nonneoplastic solid lesions of the pancreas. Radiographics 2011;31:993-1015.

56. Debray MP, Geoffroy O, Laissy JP, et al. Imaging appearances of metastases from neuroendocrine tumours of the pancreas. Br J Radiol 2001;74:1065-70.

57. Armstrong EA, Beal EW, Shah M, et al. Radiographic characteristics of neuroendocrine liver metastases do not predict clinical outcomes following liver resection. Hepatobiliary Surg Nutr 2020;9:1-12. 
58. Gibril F, Doppman JL, Reynolds JC, et al. Bone metastases in patients with gastrinomas: a prospective study of bone scanning, somatostatin receptor scanning, and magnetic resonance image in their detection, frequency, location, and effect of their detection on management. J Clin Oncol 1998;16:1040-53.

59. Lin XZ, Wu ZY, Tao R, et al. Dual energy spectral CT imaging of insulinoma-Value in preoperative diagnosis compared with conventional multi-detector CT. Eur J Radiol 2012;81:2487-94.

60. Xue HD, Liu W, Xiao Y, et al. Pancreatic and peripancreatic lesions mimic pancreatic islet cell tumor in multidetector computed tomography. Chin Med J (Engl) 2011;124:1720-5.

61. Addeo G, Beccani D, Cozzi D, et al. Groove pancreatitis: a challenging imaging diagnosis. Gland Surg 2019;8:S178-87.

62. Demos TC, Posniak HV, Harmath C, et al. Cystic lesions of the pancreas. AJR Am J Roentgenol 2002;179:1375-88.

Cite this article as: Bicci E, Cozzi D, Ferrari R, Grazzini G, Pradella S, Miele V. Pancreatic neuroendocrine tumours: spectrum of imaging findings. Gland Surg 2020;9(6):2215-2224. doi: $10.21037 / g s-20-537$
63. Kawamoto S, Johnson PT, Hall H, et al. Intrapancreatic accessory spleen: CT appearance and differential diagnosis. Abdom Imaging 2012;37:812-27.

64. Zhang L, Liao Q, Hu Y, et al. Paraganglioma of the pancreas: a potentially functional and malignant tumor. World J Surg Oncol 2014;12:218.

65. Raman SP, Hruban RH, Cameron JL, et al. Pancreatic imaging mimics: part 2, pancreatic neuroendocrine tumors and their mimics. AJR Am J Roentgenol 2012;199:309-18.

66. Srisajjakul S, Prapaisilp P, Bangchokdee S. CT and MR features that can help to differentiate between focal chronic pancreatitis and pancreatic cancer. Radiol Med 2020;125:356-64.

67. Egorov AV, Vasilyev IA, Musayev GH, et al. The role of microwave ablation in management of functioning pancreatic neuroendocrine tumors. Gland Surg 2019;8:766-72.

68. Öberg K. Management of functional neuroendocrine tumors of the pancreas. Gland Surg 2018;7:20-7. 